\title{
Caracterização e perfil do desenvolvimento de crianças em puericultura de uma Estratégia de Saúde da Família
}

\author{
Bibiana Mafaldo Consi, Alessandra Bombarda Müller
}

\section{RESUMO}

Objetivo: identificar o perfil de desenvolvimento de crianças assistidas em consultas de puericultura de uma Estratégia de Saúde da Família e os fatores que influenciam esse desenvolvimento.

Revista da Rede APS 2021

Método: pesquisa observacional, prospectiva, descritiva, do tipo transversal, com entrevistas semiestruturadas sobre questões socioeconômicas e ambientais. $\mathrm{O}$ instrumento de avaliação do desenvolvimento infantil foi o Survey of Well-being of Young Children (SWYC).

Publicada em: 28/12/2021

DOI:10.14295/aps.v3i3.162

Bibiana Mafaldo Consi (Unisinos, São Leopoldo, RS, Brasil)

Resultados: 26 crianças entre dois e 19 meses participaram do estudo, com maior prevalência do sexo feminino $(57,7 \%)$. A maioria das crianças da amostra não frequentava creche $(88,5 \%)$. Na avaliação dos marcos do desenvolvimento infantil, foi observado comportamento motor adequado para a faixa etária avaliada, entretanto, resultados abaixo da média foram encontrados especificamente na área da linguagem.

Conclusão: estes resultados poderão contribuir para o planejamento de ações específicas para as demandas desta população na unidade de saúde, e, por consequência, melhorar as condições de saúde destas crianças e suas famílias.

Palavras-chave: Desenvolvimento Infantil, Perfil de Saúde, Atenção Primária à Saúde, Fisioterapia, Diagnóstico da Situação de Saúde.

Alessandra Bombarda Müller (Unisinos, São Leopoldo, RS, Brasil)

Correspondência para:

Alessandra Bombarda Müller abombarda@unisinos.br

\section{ABSTRACT}

Objective: to identify the development profile of children assisted in childcare consultations of a Family Health Strategy and the factors that influence this development.

Method: observational, prospective, descriptive, cross-sectional research, with semi-structured interviews on socioeconomic and environmental issues. The instrument for assessing child development was the Survey of Well-being of Young Children (SWYC).

Results: 26 children between two and 19 months participated in the study, with a higher prevalence of females (57.7\%). Most children in the sample did not attend daycare (88.5\%). In the assessment of child development milestones, adequate motor behavior was observed for the age group evaluated, however, below-average results were found specifically in the area of language.

Conclusion: these results may contribute to the planning of specific actions for the demands of this population in the health unit, and, consequently, to improve the health conditions of these children and their families.

Keywords: Child Development. Health Profile. Primary Health Care. Physical Therapy Speciality. Diagnosis of Health Situation.

Submissão recebida em 20 de dezembro de 2020.

Aceito para publicação em 09 de dezembro de 2021.

Avaliado pelo sistema Double Blind Review 


\section{INTRODUÇÃO}

O direito à saúde como dever do Estado atualmente está garantido à população brasileira por meio do Sistema Único de Saúde (SUS), que propõe o acesso universal, integral, igualitário e intersetorial às ações e serviços de promoção, proteção e recuperação da saúde, sendo as ações preventivas uma de suas prioridades. Considerando esse conceito, é papel do fisioterapeuta, uma vez que o código de ética deste profissional estabelece como uma de suas funções a atuação com caráter de prevenção, a promoção da saúde e prevenção de agravos (BRASIL, 2009).

A Estratégia de Saúde da Família (ESF), desde a sua criação em 1993, vem se consolidando como um dos eixos estruturantes do SUS, por meio do movimento de expressiva expansão de cobertura populacional, aprimorando em muito o acesso da população às ações de saúde ${ }^{1}$. Nesta Estratégia, um dos instrumentos utilizados para o acompanhamento da saúde das crianças é o Programa de Puericultura, que tem como propósito acompanhar o crescimento e desenvolvimento, observar a cobertura vacinal, estimular a prática do aleitamento materno, orientar a introdução da alimentação complementar e prevenir as doenças que mais frequentemente acometem as crianças no primeiro ano de vida (LEITE, BERCINI, 2005).

A Política Nacional de Prevenção de Deficiências (BRASIL, 2010) descreve que prevenir implica realizar ações que impeçam a ocorrência de fatos ou fenômenos prejudiciais à vida e à saúde e, caso ocorram, evitar a progressão de seus efeitos. Assim, quanto mais ações preventivas, menor o impacto no desenvolvimento e o risco biopsicossocial para a criança. O fisioterapeuta tem competência para atuar na Atenção Básica por meio da educação, prevenção e assistência coletiva, bem como integrar equipes multiprofissionais destinadas a planejar, programar, controlar e executar projetos e programas (COFFITO, 2005). Uma de suas atuações pode ser no acompanhamento das Submissão recebida em 20 de dezembro de 2020 . Aceito para publicação em 09 de dezembro de 2021. Avaliado pelo sistema Double Blind Review consultas de puericultura, verificando regularmente os registros da Caderneta de Saúde da criança com o objetivo de promover a vigilância à saúde infantil. Elaborada pelo Ministério da Saúde, esta caderneta é uma importante ferramenta que os profissionais da saúde dispõem para acompanhar a adequação do crescimento e do desenvolvimento (BRASIL, 2013).

O desenvolvimento infantil é parte fundamental do desenvolvimento humano que acontece nos primeiros anos de vida, onde é estruturada a arquitetura cerebral a partir da interação entre herança genética e influências do meio em que a criança vive. As condições ambientais favoráveis e o entendimento dos cuidadores sobre as características e necessidades da criança neste processo favorecem $O$ seu desenvolvimento global (MÜLLER, SACCANI, VALENTINI, 2017). Desde o nascimento, os bebês interagem por meio de um repertório comportamental que inclui movimentos espontâneos e respostas automáticas - as primeiras manifestações que constituem o comportamento motor humano. Incentivar a aquisição de habilidades motoras nos bebês auxiliará na qualidade das interações e expandirá suas oportunidades no ambiente em que estão inseridos (SACCANI, VALENTINI, 2012).

Considerando que na primeira infância ocorrem diversos eventos neurofisiológicos relacionados ao crescimento e desenvolvimento de órgãos e tecidos corporais e que perturbações externas podem afetar este processo, torna-se essencial a identificação precoce de crianças em risco. A partir desta identificação, pode-se realizar intervenções mais rápidas, de menor custo e mais efetivas comparadas a outras fases da vida, devido à grande plasticidade cerebral e ao desenvolvimento fisiológico das crianças (MÜLLER, SACCANI, VALENTINI, 2017; SACCANI, VALENTINI, 2012).

A fisioterapia, portanto, tem um papel fundamental no desenvolvimento da criança, 
auxiliando sua organização global, identificando alterações, se presentes, orientando os pais, assim possibilitando a compensação dos fatores de risco para atraso (CAVALCANTE, 2017). A vigilância do desenvolvimento é um processo contínuo de acompanhamento das atividades relacionadas à promoção do potencial de desenvolvimento da criança e à detecção de problemas (BRASIL, 2013). Cada criança é um ser único, que deve ser entendido, cuidado e respeitado. Seu crescimento e desenvolvimento são importantes indicadores de saúde, sendo fundamental estimulá-los desde cedo para que adquira autoconfiança, autoestima e desenvolva a capacidade de se relacionar bem com a família, outras crianças e comunidade.

No contexto da importância da avaliação do desenvolvimento infantil prevista durante as consultas de puericultura na Atenção Básica, este estudo teve como objetivo identificar o perfil de desenvolvimento de crianças assistidas em consultas de puericultura de uma Estratégia de Saúde da Família (ESF), bem como os fatores que influenciam esse desenvolvimento.

\section{MÉTODO}

Estudo observacional, prospectivo, descritivo, do tipo transversal, que analisou o perfil do desenvolvimento e características associadas de crianças assistidas em consultas de puericultura de uma ESF no município de Sapucaia do Sul, RS.

Inicialmente, foi realizada uma conversa com os enfermeiros da ESF e com o Diretor de Ações em Saúde do município para expor os objetivos da pesquisa. Após a autorização para a realização do estudo e assinatura da carta de anuência pelo município, o projeto foi submetido ao Comitê de Ética e Pesquisa da instituição proponente, sendo aprovado no mês de Junho de 2020.

Considerando a situação atual que envolve o novo Coronavírus (COVID-19), o estudo foi realizado por meio de contato telefônico com os responsáveis para evitar exposição da criança e familiares no serviço de saúde. Os dados das crianças selecionadas estavam registrados em uma planilha na unidade de saúde. Foram incluídas todas as crianças que já realizavam acompanhamento prévio e se enquadravam na idade entre um a 24 meses. Foram excluídas aquelas crianças cujos responsáveis não responderam a entrevista.

A coleta de dados foi realizada nos meses de Agosto e Setembro de 2020 e a concordância daqueles responsáveis que aceitaram responder as perguntas por telefone configurou 0 consentimento na participação na pesquisa. Após a entrevista semiestruturada elaborada pela pesquisadora, onde foram pesquisados dados como idade gestacional ao nascimento, tempo de amamentação exclusiva, se a criança frequenta escola de educação infantil, nível de escolaridade dos pais, entre outros, foi aplicado o questionário Survey of Well-being of Young Children (SWYC) (PERRIN et al., 2016), mundialmente utilizado, já validado nacionalmente e indicado pelo Ministério da Saúde. É um instrumento de vigilância do desenvolvimento infantil de rápida e fácil aplicação (em média, 10 minutos), o que o torna viável para utilização na atenção primária à saúde.

O SWYC foi desenvolvido para a faixa etária de um a 65 meses e não requer um kit específico. Está disponível online, sem qualquer custo, para as famílias e outros profissionais envolvidos com o cuidado na primeira infância. É dividido em três grandes domínios que permitem obter informações não somente dos marcos do desenvolvimento, mas também sobre o comportamento e os fatores de risco da família que podem estar contribuindo para adversidades no desenvolvimento, como a depressão e a violência doméstica, dentre outros (PERRIN et al., 2016).

As informações coletadas foram tabeladas em ficha online no Microsoft Office Excel versão 2011, para padronização das informações. Os dados foram agrupados, sintetizados e organizados de forma esquematizada no processador estatístico Statistical Package for the Social Science (SPSS), versão 22.0. Foi realizada análise descritiva dos dados, utilizando distribuição de frequência e porcentagem para as variáveis categóricas e medidas de tendência 
central para as contínuas. Após a coleta dos dados, todos os responsáveis pelas crianças participantes receberam, via WhatsApp, documentos norteadores para auxiliar no estímulo do desenvolvimento infantil no domicílio pelos familiares.

\section{RESULTADOS}

A amostra deste estudo, por conveniência, foi composta por crianças entre dois e 19 meses que já realizavam acompanhamento em consultas de puericultura na ESF. Inicialmente, 59 crianças identificadas preenchiam os critérios de inclusão para esta pesquisa, entretanto, apenas 26 participaram, cujos responsáveis atenderam as ligações telefônicas e aceitaram responder à entrevista.

A tabela 1 apresenta a caracterização geral da amostra. Quanto aos dados referentes às crianças, foi verificada prevalência do sexo feminino ( $n=15,57,7 \%)$, média de idade $9,18 \pm 6,00$ meses, cor branca $(n=21,80,8 \%)$ e $88,5 \%$ das crianças não frequentavam escola de educação infantil $(n=23)$. Quanto às características maternas das crianças avaliadas, $42,3 \%$ das mães $(n=11)$ possuíam o ensino médio completo e a mesma proporção tinha como ocupação ser dona de casa.

Tabela 1 - Caracterização geral da amostra $(n=26)$

\begin{tabular}{|c|c|}
\hline Variáveis & amostra \\
\hline 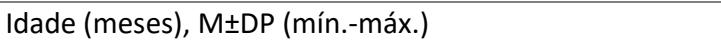 & $9,18 \pm 6,00(2-19)$ \\
\hline 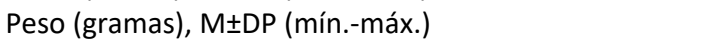 & $3081,52 \pm 538,66(2100-4460)$ \\
\hline Idade gestacional (semanas), M $\pm D P$ (mín.-máx.) & $38 \pm 1,84(36-40)$ \\
\hline \multicolumn{2}{|l|}{ Sexo, n (\%) } \\
\hline feminino & $15(57,7)$ \\
\hline masculino & $11(42,3)$ \\
\hline \multicolumn{2}{|l|}{ Cor, n (\%) } \\
\hline branca & $21(80,8)$ \\
\hline parda & $4(15,4)$ \\
\hline preta & $1(3,8)$ \\
\hline \multicolumn{2}{|l|}{ Aleitamento materno, $\mathrm{n}(\%)$} \\
\hline $\operatorname{sim}$ & $17(65,4)$ \\
\hline não & $9(34,6)$ \\
\hline complementar & $7(41,2)$ \\
\hline exclusivo & $5(29,4)$ \\
\hline predominante & $5(29,4)$ \\
\hline \multicolumn{2}{|l|}{ Frequenta escola de educação infantil, n (\%) } \\
\hline não & $23(88,5)$ \\
\hline $\operatorname{sim}$ & $3(11,5)$ \\
\hline \multicolumn{2}{|l|}{ Escolaridade da mãe, n (\%) } \\
\hline fundamental incompleto & $3(11,5)$ \\
\hline médio incompleto & $4(15,4)$ \\
\hline médio completo & $11(42,3)$ \\
\hline superior incompleto & $3(11,5)$ \\
\hline superior completo & $5(19,2)$ \\
\hline \multicolumn{2}{|l|}{ Ocupação da mãe, n (\%) } \\
\hline do lar & $11(42,3)$ \\
\hline cabeleireira & $3(11,5)$ \\
\hline outras & $12(46,2)$ \\
\hline Renda familiar, M $\pm D P$ (mín.-máx.) & $2 \pm 1,21(1-6)$ \\
\hline \multicolumn{2}{|l|}{ Benefício incremento renda, $\mathrm{n}(\%)$} \\
\hline nenhum & $14(53,8)$ \\
\hline auxílio emergencial & $7(26,9)$ \\
\hline PBF & $5(19,2)$ \\
\hline 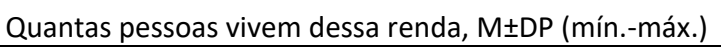 & $4 \pm 1,31(2-6)$ \\
\hline
\end{tabular}

*Resultados expressos por média (M), desvio padrão (DP), valores mínimos (mín.) e máximos (máx.) e proporção (\%).

Fonte: Elaborada pelas autoras. 
A tabela 2 apresenta alguns dos marcos do desenvolvimento infantil questionados no instrumento da pesquisa e o número absoluto e percentual de crianças que realizavam as habilidades na faixa etária preconizada, onde verifica-se pior desempenho nas habilidades associadas à área da linguagem com o passar do tempo.

Tabela 2 - Marcos do desenvolvimento ( $n=26$ crianças)

\begin{tabular}{|c|c|c|c|c|c|c|c|c|c|c|}
\hline \multirow[t]{2}{*}{ Habilidades } & \multicolumn{10}{|c|}{$\mathrm{n}(\%)$ meses } \\
\hline & $2-4$ & $2-6$ & $4-6$ & $6-9$ & $6-12$ & $9-15$ & $12-18$ & $15-18$ & $15-24$ & $18-24$ \\
\hline Acompanha o brinquedo & $\begin{array}{c}6 \\
(100,0)\end{array}$ & & & & & & & & & \\
\hline Controla a cabeça e mãos & $\begin{array}{c}10 \\
(90,9)\end{array}$ & & & & & & & & & \\
\hline Balbucia & & $\begin{array}{c}13 \\
(92,9)\end{array}$ & & & & & & & & \\
\hline Fica em prono & & & $\begin{array}{c}4 \\
(50,0)\end{array}$ & & & & & & & \\
\hline Transfere o objeto & & & $\begin{array}{c}4 \\
(50,0)\end{array}$ & & & & & & & \\
\hline Senta-se sozinho & & & & $\begin{array}{c}5 \\
(71,4)\end{array}$ & & & & & & \\
\hline Leva o alimento à boca & & & & & $\begin{array}{c}5 \\
(62,5)\end{array}$ & & & & & \\
\hline Puxa para ficar em pé & & & & & $\begin{array}{c}8 \\
(100,0)\end{array}$ & & & & & \\
\hline $\begin{array}{l}\text { Brinca de esconde- } \\
\text { esconde }\end{array}$ & & & & & $\begin{array}{c}5 \\
(100,0)\end{array}$ & & & & & \\
\hline Chama "mama" ou "papa" & & & & & & $\begin{array}{c}8 \\
(100,0)\end{array}$ & & & & \\
\hline Imita sons & & & & & & $\begin{array}{c}7 \\
(87,5)\end{array}$ & & & & \\
\hline Marcha independente & & & & & & $\begin{array}{c}7 \\
(87,5)\end{array}$ & & & & \\
\hline Obedece a ordens simples & & & & & & $\begin{array}{c}8 \\
(100,0)\end{array}$ & & & & \\
\hline Corre & & & & & & & $\begin{array}{c}7 \\
(87,5)\end{array}$ & & & \\
\hline Sobe escadas com auxílio & & & & & & & $\begin{array}{c}7 \\
(87,5)\end{array}$ & & & \\
\hline Chuta a bola & & & & & & & & $\begin{array}{c}5 \\
(83,3)\end{array}$ & & \\
\hline Nomeia partes do corpo & & & & & & & & & $\begin{array}{c}2 \\
(28,6)\end{array}$ & \\
\hline $\begin{array}{l}\text { Sobe escadas } \\
\text { independente }\end{array}$ & & & & & & & & & & $\begin{array}{c}1 \\
(25,0)\end{array}$ \\
\hline Pula com os dois pés & & & & & & & & & & $\begin{array}{c}3 \\
(75,0)\end{array}$ \\
\hline Pede ajuda & & & & & & & & & & $\begin{array}{c}1 \\
(25,0)\end{array}$ \\
\hline
\end{tabular}

*Resultados expressos por número absoluto (n) e proporção (\%).

Fonte: Elaborada pelas autoras.

A tabela 3 apresenta algumas das características socioemocionais das crianças e suas cuidadoras (mães), bem como questões de cuidado e rotina familiar. Identificar estas questões, principalmente logo após a chegada do novo bebê, pode promover o manejo mais adequado da criança e da sua família, facilitando a adaptação do bebê, o reconhecimento das suas necessidades e a melhor interação mãe-bebê. Ao analisar as mães de crianças com idade até 
seis meses, 35,7\% $(n=5)$ relataram sentir-se, na maioria das vezes, culpadas sem necessidade quando as coisas não corriam bem, 50\% sentiam-se, na maioria das vezes, ansiosas ou preocupadas sem motivo, $41,7 \%$ falaram estar, na maioria das vezes, com medo ou muito assustadas sem grande motivo, e uma mãe relatou já ter tido ideias de fazer mal a si mesma.

Tabela 3 - Características socioemocionais das crianças e suas mães $(n=26)$

\begin{tabular}{|c|c|c|c|c|}
\hline \multirow[t]{2}{*}{ Questões } & \multicolumn{3}{|c|}{$\mathrm{n}(\%)$ meses } & \multirow[b]{2}{*}{$2-24$} \\
\hline & $2-15$ & $18-24$ & $2-6$ & \\
\hline Fica incomodada com pessoas estranhas e diferentes lugares & $7(31,8)$ & & & \\
\hline Chora muito & $2(9,1)$ & & & \\
\hline Difícil acalmar & $11(50,0)$ & & & \\
\hline Se irrita fácil & $9(40,9)$ & & & \\
\hline Dificuldade na rotina diária & $7(31,8)$ & & & \\
\hline Dificuldade para dormir & $4(18,2)$ & & & \\
\hline Parece medrosa ou nervosa & & $1(25,0)$ & & \\
\hline Parece triste ou infeliz & & 0 & & \\
\hline Dificuldades com mudanças na rotina & & $1(25,0)$ & & \\
\hline Dificuldades para brincar com outras crianças & & $1(25,0)$ & & \\
\hline Dificuldades para prestar atenção & & $1(25,0)$ & & \\
\hline Agressiva / inquieta & & $2(50,0)$ & & \\
\hline Brava ou zangada & & $3(75,0)$ & & \\
\hline Sabe o que a criança necessita & & $1(25,0)$ & & \\
\hline Mantém a rotina diária & & $4(100,0)$ & & \\
\hline A criança obedece e interage com você & & $2(50,0)$ & & \\
\hline Brincadeiras favoritas (bonecos / bichos de pelúcia) & & $3(75,0)$ & & \\
\hline Fica chateada quando as coisas não são feitas do seu jeito & & $4(100,0)$ & & \\
\hline A criança mostra o que quer somente apontando com o dedo? & & $3(75,5)$ & & \\
\hline Sente-se culpada quando as coisas não ocorrem bem? & & & $5(35,7)$ & \\
\hline Sente-se ansiosa ou preocupada sem motivo? & & & $7(50,0)$ & \\
\hline Sente-se com medo ou muito assustada sem grande motivo? & & & $5(41,7)$ & \\
\hline Já teve ideias de fazer mal a si mesma? & & & $1(7,9)$ & \\
\hline Nos últimos 7 dias, alguém leu para a criança todos os dias? & & & & $4(15,4)$ \\
\hline
\end{tabular}

*Resultados expressos por número absoluto (n) e proporção (\%).

Fonte: Elaborada pelas autoras.

\section{DISCUSSÃO}

O objetivo deste estudo foi identificar o perfil do desenvolvimento e características associadas de crianças assistidas previamente em consultas de puericultura de uma ESF. Avaliar o desenvolvimento infantil é uma tarefa complexa, que exige uma vigilância continuada nos primeiros anos de vida e o conhecimento do comportamento esperado para as suas diferentes fases. Apesar de haver inúmeros instrumentos de triagem, não existe um que tenha uma abrangência universal. Além disso, a maioria dos instrumentos existentes exigem treinamento, tempo prolongado para realização e interpretação, além de recursos materiais e financeiros. Isso traz como consequência o pouco uso de instrumentos padronizados de avaliação do desenvolvimento infantil na prática clínica (MARKS, LAROSA, 2012).

Por essas questões, foi utilizado para esta pesquisa o SWYC, um questionário norteamericano criado em 2011 e validado em 2013 por Perrin e colaboradores, reconhecido como uma ferramenta de triagem de atraso no desenvolvimento de rápida e fácil aplicação, podendo ser administrado pessoalmente, por computador ou telefone. Este instrumento fornece uma visão global da criança por meio de vigilância continuada, utilizando questionários 
para acompanhar o progresso do desenvolvimento, do comportamento, além do contexto familiar da criança. Sua aplicação não requer um kit específico, brinquedos ou materiais extras, e pode ser realizada em qualquer local, inclusive na atenção primária à saúde (PERRIN et al., 2016), como realizado nesta pesquisa.

Há algumas décadas a saúde da mulher e da criança têm sido mundialmente estudadas, e no Brasil são consideradas prioridades públicas, entretanto, o número de mortes decorrentes de complicações na gestação e no parto ainda é elevado (FRANÇA, LANSKY, 2008). A literatura traz que os primeiros 1.000 dias de vida compreendem desde o momento da concepção até os dois anos de idade da criança. São 270 dias da gestação, mais 365 dias do primeiro ano de vida somados aos 365 dias do segundo ano. Essa etapa da vida é crucial para o crescimento e desenvolvimento infantil, pois trata-se de um período de "janela de oportunidades", no qual é possível adotar hábitos e atitudes que irão influenciar o futuro do bebê (VICTORA et al., 2008).

Nesse panorama, o Ministério da Saúde, em 2002, ao publicar na série de Cadernos de Atenção Básica um número intitulado "Saúde da criança: acompanhamento do crescimento e desenvolvimento infantil" trouxe a proposta de atenção integral e multiprofissional à saúde da criança, observando o seu crescimento e desenvolvimento, por meio de ações na atenção primária em saúde (SCHAIK et al., 2014) a fim de garantir a qualidade da assistência prestada à criança, propõe um calendário mínimo de consultas de puericultura, assim distribuídas: uma consulta até os quinze dias de vida, consultas com 1 mês, 2, 4, 6, 12 e 18 meses, totalizando assim, sete consultas no primeiro ano e meio de vida.

A puericultura é desenvolvida tanto pelo médico quanto pelo enfermeiro. As atribuições dos profissionais neste programa são: realizar o exame físico na criança, identificando riscos em seu crescimento e desenvolvimento; agendar consulta com especialista quando forem identificados riscos de agravos à saúde; fornecer a relação dos nascidos vivos para os Agentes Comunitários de Saúde (ACS) e solicitar a busca ativa para identificação dos faltosos do programa; preencher o gráfico de peso e estatura nos cartões da criança; verificar e administrar as vacinas conforme o calendário básico de vacinação; incentivar o aleitamento materno exclusivo até os seis meses; orientar a alimentação complementar após os seis meses; orientar sobre prevenção de acidentes de acordo com a faixa etária; avaliar o desenvolvimento neuropsicomotor; identificar dúvidas e dificuldades da mãe e de outros membros da família que participam das consultas, procurando esclarecê-las (VIEIRA et al., 2012).

Em relação ao tempo de aleitamento materno, $65,4 \%$ das crianças desta amostra ( $n=17)$ ainda estavam sendo amamentadas, dado que corrobora estudos que trazem a associação do maior tempo de amamentação com melhores resultados no desenvolvimento motor, podendo ser explicada pela interação de qualidade da díade mãe-bebê que é concedida com o ato do aleitamento materno, agindo assim como fator de proteção para o desenvolvimento da criança (MÜLLER, SACCANI, VALENTINI, 2017; SACCANI, VALENTINI, 2012).

A necessidade de garantir o pleno desenvolvimento infantil fica mais evidente nos países onde as crianças são expostas a fatores de risco como desnutrição, pobreza, violência, ambientes pouco estimulantes, dentre outros. Estima-se que mais de 200 milhões de crianças abaixo dos cinco anos provenientes de países em desenvolvimento, como o Brasil, não conseguirão atingir todo seu potencial de desenvolvimento. Na mesma direção, no presente estudo, $46,2 \%$ dos cuidadores relataram possuir renda familiar média de 2 salários-mínimos, e 53,8\% não recebiam benefício de incremento de renda, o que pode impactar nos fatores de alimentação, ambiente, lazer e, automaticamente, no desenvolvimento infantil (GRANTHAM-MCGREGOR et al., 2007).

De acordo com o DATASUS, no ano de 2018 nasceram 894 bebês do sexo masculino e 853 do sexo feminino na cidade de Sapucaia do Sul, 
números aproximados que confirmam os achados do presente estudo, cuja amostra foi semelhante entre os sexos. Foi possível observar que $80,8 \%(n=21)$ das crianças analisadas eram da cor branca. Segundo o Instituto Brasileiro de Geografia e Estatística, no ano de 2016 a população residente do Brasil foi estimada em 205,5 milhões de pessoas, revelando que no critério relacionado à cor ou raça, a maioria da população brasileira é parda $(46,7 \%)$ ou branca (44,2\%). Na Região Sul, $76,8 \%$ se declaram brancas e $18,7 \%$ pardas, o que corrobora os achados deste estudo, sendo que a coleta foi realizada na Região do Vale do Rio dos Sinos, onde a maioria da população é descendente de alemães.

Entre os fatores ambientais, a baixa renda familiar e o baixo grau de escolaridade dos pais são fatores comumente associados a resultados desfavoráveis para o desenvolvimento de crianças em geral. Estudo prévio com 239 lactentes com idade entre três e 18 meses revelou que a maior escolaridade dos pais e o maior nível socioeconômico foram associados às melhores oportunidades de estimulação motora no lar, influenciando positivamente o desenvolvimento motor destas crianças (DEFILIPO et al., 2012).

Quanto à escolaridade, pode-se observar que $42,3 \%$ das mães ( $n=11)$ possuem o ensino médio completo. Outros estudos apontam a escolaridade materna como um fator determinante para o crescimento e desenvolvimento infantil, ressaltando que uma mãe com maior escolaridade pode contribuir positivamente para o desenvolvimento por oferecer um ambiente de qualidade ao seu filho (NEVES et al., 2016).

A depressão materna é um fator de risco bem determinado na literatura e potencialmente modificável quando oportunizada uma intervenção precoce. O uso de um instrumento para sua detecção precoce é necessário, além da informação sobre tratamento psicoterápico e/ou medicamentoso (MARKS, LAROSA, 2012).

Durante a pesquisa foi identificada uma mãe que relatou sentir vontade de fazer mal a si mesma. Cantilino e colaboradores (2010), apontam que o período do pós-parto é considerado o de maior vulnerabilidade para o aparecimento de transtornos psiquiátricos. Entre eles encontram-se a disforia puerperal, a depressão pós-parto, a psicose pós-parto e os transtornos ansiosos. Dessa forma, por ser um período diferenciado da vida, é importante conhecer quais os fatores podem evitar ou contribuir com os eventos estressantes relacionados à gravidez e ao puerpério para que estratégias psicossociais sejam planejadas a fim de minimizar o impacto de sintomatologias psicológicas/psiquiátricas na relação mãe-bebê, inclusive, nas relações familiares (BAPTISTA, BAPTISTA, TORRES, 2006).

As práticas parentais de cuidado com a criança, quando adequadas às necessidades do desenvolvimento infantil, têm impacto tanto no desempenho motor como também nas habilidades de comunicação e no comportamento social da criança, desde a infância até a fase adulta. Estudo recente relatou haver relação das práticas parentais e o surgimento dos marcos motores no primeiro ano de vida, a partir de quatro meses de idade, e as práticas relacionadas com maior oferta de material para aprendizagem e liberdade de movimento foram as mais favoráveis ao desenvolvimento (RIBEIRO et al., 2018).

Pesquisas prévias confirmam estes resultados ao afirmarem que o envolvimento dos pais, associado ao adequado espaço físico da casa e a uma oferta variada de brinquedos lúdicos, permitirão que a criança alcance todo seu potencial de desenvolvimento (MÜLLER, SACCANI, VALENTINI, 2017; SOUZA, VERÍSSIMO, 2015; CORREA et al., 2019). Portanto, as práticas de qualidade oferecidas à criança se mostram associadas positivamente ao desenvolvimento cognitivo e da linguagem, com evidências do impacto dessa prática adequada realizada na primeira infância, com repercussão na idade escolar (BURCHINAL et al., 2000).

A família é o primeiro meio estimulador dentro do processo de desenvolvimento, cujas relações entre seus membros são caracterizadas como íntimas e intergeracionais, onde os pais, desde o nascimento de seus filhos, estimulam seu 
desenvolvimento social, afetivo, cognitivo e comportamental (CORREA et al., 2019; BLACK et al., 2008).

Juntamente com a aquisição das habilidades motoras e cognitivas, a criança desenvolve suas habilidades de linguagem e comunicação ao longo dos primeiros dois anos de vida. Então, percebe-se que a linguagem receptiva se desenvolve antes da expressiva, uma vez que as crianças entendem antes de serem capazes de falar (GOLDIM-MEADOW, 2007). Além disso, não é raro ver uma criança dessa idade pedir alguma coisa utilizando a combinação de gestos e sons. A linguagem gestual faz parte do processo evolutivo da linguagem, que se desenvolve próximo do final do primeiro ano (ROSS, DEMARIA, YAP, 2018), validando o resultado deste trabalho, onde $75 \%$ das crianças apontavam o dedo para mostrar o que queriam, segundo o relato das mães. É importante destacar que o ambiente doméstico é um local potencialmente significativo para gerar oportunidades de estimulação do desenvolvimento infantil (BATENBURG-EDDES et al., 2013).

No presente estudo, as habilidades associadas ao domínio da linguagem apresentaram-se deficitárias conforme o crescimento da criança. Estudo prévio afirma que alterações na trajetória motora de crianças dos dois aos quatro meses foram associadas com atrasos na linguagem receptiva e expressiva aos 1,5 e 2,5 anos de idade, confirmando a interação do comprometimento da função motora nos estágios iniciais da trajetória do desenvolvimento infantil e o surgimento subsequente de atrasos na cognição e linguagem (BATENBURG-EDDES et al., 2013). Outro estudo refere que o desempenho cognitivo contribuiu significativamente para a variação dos escores de linguagem receptiva; enquanto o desempenho motor teve relação com $o$ desenvolvimento da linguagem expressiva. Esses resultados sugerem que as áreas cerebrais do controle motor podem interagir no desenvolvimento da linguagem de crianças (ROSS, DEMARIA, YAP, 2018).
Ainda entre os fatores relacionados ao ambiente, o conhecimento dos pais acerca do desenvolvimento infantil e do cuidado que a criança demanda pode determinar a maneira como os pais educam seus filhos (CORREA et al., 2019). Ao final das entrevistas, foi enviado material educativo para as mães que participaram da pesquisa com intuito de auxiliar e esclarecer os marcos motores e como pode ser feita a estimulação no âmbito familiar, pois é consenso que as mães que apresentam maior conhecimento a respeito do desenvolvimento infantil tendem a estabelecer interações menos conflituosas com a criança, por terem expectativas e percepções mais reais a respeito do próprio comportamento, das condutas e necessidades de seu filho (BARTLETT et al., 2011).

As habilidades motoras ao longo do desenvolvimento infantil aparecem em uma sequência definida e em um período relativamente previsível, porém em ritmos distintos entre as crianças, devido às restrições do indivíduo, que mudam rapidamente, tanto estruturais quanto funcionais, do ambiente e da tarefa (MÜLLER, SACCANI, VALENTINI, 2017). O desenvolvimento é um comportamento com períodos de estabilidade e variabilidade durante todo o processo. Muitas vezes, as crianças não apresentam padrão linear ao longo dos meses, observando-se períodos de maior e menor estabilidade na aquisição de habilidades motoras e com semelhanças no desempenho motor entre os sexos, com curvas de desenvolvimento das aquisições posturais em prono, supino, sentado e em pé em padrão não linear, com maior número de aquisições entre o segundo e terceiro trimestres (SACCANI, VALENTINI, 2012).

A experiência de ler conjuntamente com um bebê, observando as mesmas figuras, funciona como uma poderosa situação de aprendizagem para a criança, onde duas pessoas se relacionam, compartilham significados e podem pensar juntas (SOUZA, VERÍSSIMO, 2015). O olhar compartilhado que os livros oferecem transforma-se em um potente instrumento de pensamento e sensibilidade para as relações humanas, como preconiza o Ministério da 
Educação. Os resultados do presente estudo constatam que $57,7 \%$ das mães não leem para seus filhos. Nesse sentido, é importante orientar as mães sobre a importância da leitura, pois ler para um bebê é um momento também de criar vínculo afetivos, importante para seu desenvolvimento.

Este estudo apresenta limitações como a dificuldade em contatar algumas famílias adscritas. Muitas vezes, elas não permaneciam com o mesmo número telefônico, não atendiam ou deixavam o aparelho desligado, além do cadastro desatualizado. Apesar dos profissionais tentarem manter os dados atualizados, muitos usuários trocam de endereço ou acessam a unidade eventualmente, comprometendo os resultados encontrados.

\section{CONCLUSÃO}

Esta análise das crianças assistidas em consultas de puericultura de uma ESF do município de Sapucaia do Sul identificou o perfil desta população: meninos e meninas de até dois anos de idade, de cor branca, com prevalência de nascimento a termo e que não frequentam escola de educação infantil, provavelmente em decorrência das restrições sociais como consequência da pandemia que estamos vivendo. Os resultados também apontam uma grande influência dos cuidados maternos no desenvolvimento destas crianças, que reforça a necessidade dos responsáveis e cuidadores obterem informações básicas sobre o desenvolvimento infantil para minimizar possíveis atrasos, se presentes.

A maior parte das crianças apresentou comportamento motor satisfatório para a faixa etária, entretanto, resultados pouco expressivos foram encontrados especificamente na área da linguagem. As pesquisas prévias comprovam que estímulos adequados proporcionados pelos cuidadores podem impactar positivamente no desenvolvimento infantil, sendo fundamental o acompanhamento nas consultas de puericultura para orientações e esclarecimentos dos profissionais de saúde, bem como se aconselha que a mãe tenha suporte familiar para não ficar sobrecarregada nos cuidados com a criança.

Ressalta-se a importância do incentivo para um melhor desempenho nas aquisições motoras, através da exploração do meio, o que engloba brinquedos, alimentos, sons, mudanças de posturas, relação com os familiares, e cultura do local em que vive. Estes resultados poderão contribuir para o planejamento de ações específicas para as demandas desta população na unidade de saúde, e, por consequência, melhorar as condições de saúde destas crianças e suas famílias. 


\section{REFERÊNCIAS}

BAPTISTA M.N.; BAPTISTA A.S.D.; TORRES E.C.R. Associação entre suporte social, depressão e ansiedade em gestantes. Psic: Revista da Vetor Editora. 2006; 7(1): 39-48.

BARTLETT D., et al. Perceptions of vulnerability and variations in childrearing practices of parents of infants born preterm. Pediatric Physical Therapy. 2011; 23(3): 280-288.

BATENBURG-EDDES T., et al. Early infant neuromotor assessment is associated with language and nonverbal cognitive function in toddlers. Journal of Developmental \& Behavioral Pediatrics. 2013; 34(5): 326-334.

BLACK R., et al. Maternal and child undesnutrition: global and regional exposures and health consequences. The Lancet. 2008; 371(9608): 243-260.

BURCHINAL M., et al. Relating quality of center-based child care to early cognitive and language development longitudinally. Child Development. 2000; 71(2): 339-357.

CANTILINO A., et al. Transtornos psiquiátricos no pós-parto. Archives of Clinical Psychiatry (São Paulo). 2010; 37(6): 288-294.

CAVALCANTE C. Atuação da fisioterapia através da estimulação precoce em bebês prematuros. Rev. Eletrôn. Atualiza Saúde. 2017; 5(5): 29-36.

Conselho Federal de Fisioterapia e Terapia Ocupacional (Brasil). Saúde da Família: uma nova opção para o trabalho do fisioterapeuta e terapeuta ocupacional. Revista trimestral do COFFITO. 2005; 7(24): 6-8.

CORREA W., et al. Parental beliefs on child development of children with developmental delays. Paidéia. 2019; 29: 1-9.

DEFILIPO E.C., et al. Oportunidades do ambiente domiciliar para o desenvolvimento motor. Revista Saúde Pública. 2012; 46(4): 633-641.

FRANÇA E.; LANSKY S. Mortalidade Infantil Neonatal no Brasil: Situação, Tendências e Perspectivas. Informe de Situação e Tendências: Demografia e Saúde. 2008.

GRANTHAM-McGREGOR S., et al. Developmental potential in the first 5 years for children in developing countries. The Lancet. 2007; 369(9555): 60-70.

GOLDIM-MEADW S. Pointing sets the stage for learning language and creating language. Child Development. 2007; 78: 741-745.

LEITE G.M.; BERCINI L.O. Caracterização das crianças atendidas na puericultura do programa saúde da família do município de Campo Mourão, Paraná, em 2003. Ciência, Cuidado e Saúde. 2005; 4(3): 224-230.

MARKS K.; LAROSA A. Understanding developmental-behavioral screening measures. Pediatrics in review. 2012; 33(10): 448-457.

Ministério da Saúde (Brasil). Secretaria de Atenção à Saúde. Departamento de Atenção Básica. Saúde da criança: nutrição infantil: aleitamento materno e alimentação complementar. Brasília: Editora do Ministério da Saúde, 2009. (Série A. Normas e Manuais Técnicos) (Cadernos de Atenção Básica, n. 23). Disponível

em:

https://bvsms.saude.gov.br/bvs/publicacoes/saude crianca nutricao aleitamento alimentacao.pdf.

"Acesso em 17 de novembro de 2020." 
Ministério da Saúde (Brasil). Política Nacional de Saúde da Pessoa com Deficiência. Secretaria de Atenção à Saúde (SAS). $2010 . \quad$ Disponível em: http://bvsms.saude.gov.br/bvs/publicacoes/politica nacional pessoa com deficiencia.pdf. "Acesso em 17 de novembro de 2020."

Ministério da Saúde (Brasil). Caderneta de Saúde da Criança. Brasília. 2013. Disponível em: https://bvsms.saude.gov.br/bvs/publicacoes/caderneta saude crianca menino.pdf. "Acesso em 17 de novembro de 2020."

MÜLLER A.B.; SACCANI R.; VALENTINI N.C. Impact of compensatory intervention in 6- to 18-month-old babies at risk of motor development delas. Early Child Development and Care. 2017; 187(11): 1707-1717.

NEVES K.R., et al. Growth and development and their environmental and biological determinants. Jornal de Pediatria. 2016; 92(3): 241-250.

PERRIN E., et al. The Survey of Well-being of Young Children (SWYC) User' s Manual. 1.01 ed. Boston: Center, Tufts Medical, 2016.

RIBEIRO R.F., et al. Relação entre práticas parentais e marcos do desenvolvimento motor no primeiro ano de vida. Revista Pesquisa em Fisioterapia. 2018; 8(3): 296-304.

ROSS G.; DEMARIA R.; YAP V. The relationship between motor delays and language development in very low birthweight premature children at 18 months corrected age. Journal of Speech, Language, and Hearing Research. 2018; 61(1): 114-119.

SACCANI R.; VALENTINI N.C. Reference curves for the Brazilian Alberta Infant Motor Scale: percentiles for clinical description and follow-up over time. Jornal de Pediatria. 2012; 88(1): 40-47.

SCHAIK E.E., et al. Reflexões sobre a atenção às crianças com deficiência. Rev Ter Ocup Univ São Paulo. 2014; 25(3): 233-241.

SOUZA J.M.; VERÍSSIMO M.R. Child development: analysis of a new concept. Revista Latino-Americana de Enfermagem. 2015; 23(6): 1097-1104.

VICTORA C.G., et al. Maternal and child undesnutrition: consequences for adult health and human capital. The Lancet. 2008; 371(9609): 340-357.

VIEIRA V.C.L., et al. Puericultura na atenção primária à saúde: atuação do enfermeiro. Cogitare Enfermagem. 2012; 17(1): 119-125. 